\title{
A Smart Locker
}

\author{
D.Aswini, S.Nandakumar
}

\begin{abstract}
Nowadays, the Internet of Things is becoming a reality more and more. Beside smart thermostats, light switches and power outlets controllable by smart phone apps creating smart homes, technology has solutions for daily encountered problems as well. We have identified two specific problems related to locking system and we proposed solution for the same. The one is vehicle and the second is mail box. Having a lock on a bike or mail box is not a guarantee that the vehicle or the documents won't get stolen. The proposed work provides a convenient way of locking, providing reliable security system for the vehicle/mail box. The proposed system uses the IoT technology and smart phone to lock or unlock the device remotely through authentication. In particular, this work proposes security enhancement against any physical damage or theft. The proposed locker allows the user to lock and unlock easily and securely. Using user's phone, the lock system can be locked/unlocked by entering a pass code in the Android application. One can also use the system using the registered touch patterns that can be entered through a touch sensor present in the lock. It provides intelligent lock/unlock with the location of your phone to the locker with the Bluetooth strength. It also provides theft alert to the user.
\end{abstract}

\section{Keywords: IoT, Locker, lock on a bike, mail box}

\section{INTRODUCTION}

By 2020, bike sharing systems in the world's biggest cities are expected to offer over 2.3 million bicycles. Currently there are one million bikes in sharing/rental system. For this emerging market, smart locker plays a pivotal role in bike sharing/rental systems. Smart locker provides Bluetooth connectivity which is used to lock/unlock the device using phone. This application is also useful for tracking customer and vehicle. It is helpful at back office of the rental system provider. Back office is in turn connected to the lock's secure key server. The providers need not to have separate space for parking User has the flexibility and freedom to park the vehicle anywhere. It is more useful during peak hours. Other advantages of the smart locker is that the system stores the details of the person who has parked the bike. Also, we can able to track the vehicle.

Controlling vehicle using mobile phone is a new feature for vehicles. This feature enables the user to have remote control of vehicle like locking, unlocking, remote start and activation of an alarm. So, the vehicle manufacturers have started looking for new features to simplify and reduce the user interface for vehicle access. These features provides users with an additional level of comfort without requiring them to touch or press any button on any remote devices to gain access to the vehicle. But, on other hand, there are chances for several security threats against the vehicle's remote system.

Revised Manuscript Received on August 05, 2019.

D.Aswini, Assistant Professor, Kumaraguru College of Technology, Coimbatore, Tamilnadu, India.

(Email: aswini.d.cse@kct.ac.in)

S.Nandakumar, Assistant Professor, Kumaraguru College of Technology, Coimbatore, Tamilnadu, India.
These smart vehicles are built with higher computing power, connectivity, along with the new generation automotive design. A new generation vehicle utilizes remote keyless entry system and Immobilizer system as the main weaponry against vehicle theft. It prevents unauthorized access to the vehicle to a certain extent only. Vehicle theft incidents in worldwide are on the rise due to simple security system. So, the proposed work focuses on security aspect by incorporating a gesture based smart lock, keyless entry, touch screen ignition and adjustable motion alarm sensitivity. It can be further extended by including GPS fencing, remote fuel cut-off, vehicle tracking system and much more.

This locker can be accessed using a smartphone application, either iOS or Android. Users can lock and unlock their bikes using mobile phone. The theft alert system is activated through Bluetooth or Wi-Fi.

Smart mailbox is Wi-Fi enabled, solar powered system specifically designed for keeping the mails safe and tracking them. It secures your mail through its variety of intelligent sensors and by learning your mailman's schedule. The embedded sensors in the mail Box are able to determine when mail has arrived and lock the mailbox to protect it once it's safely inside. If you are gone for several days and get mail, the mail Box will unlock its door so mail can still be delivered and then close and lock, keeping your deliveries protected. This functionality is achieved by using data to learn the postman's schedule and create a standardized delivery window. This window can be adjusted manually or disabled for those who don't have regular delivery.

\section{EXISTING SYSTEM}

The existing system in vehicle is a Bike U-lock which uses Bluetooth Low Energy (BLE), to enable the cmmunication with the user's smartphone. On the outside, the lock has only one button to lock and unlock the bike. It has a embedded circuit and a high-power, 3.7V nonrechargeable AA battery. The battery life time is expected to be 5 years even with the wireless functionality as it needs to be on at all times to detect the approaching user. The one thing which is to be considered in U-lock is the form factor of the lock. The lock is not permanently attached to the bike and any user can open it can also steal the lock.

The existing systems in Mail Box use fingerprint based authentication system to unlock the mail box. This system allows the authorized users by verifying fingerprint. But, fingerprint sensors are expensive. Some experts also argue that only relying on a fingerprint sensor is not wise as it is relatively easy to lift someone's fingerprints and replicate 
them, which is why it is always advised to use fingerprint scanners in a two factor authentication systems where an additional layer of security is available in the form of PIN, pass code, voice recognition, etc.

Existing technologies for locking system uses physical key to lock/unlock. In case of missing of the key may leave you in an inconvenient situation. There are some alternative electronic locks available in the market. They provide any of these features like password entry, enter using phone or remote. But in our proposed system, the locker has all the above mentioned features along with the following features:

- $\quad$ Fully Automatic Locking System

- $\quad$ Secured Lock

- Keyless

- Hassle-free Locking using Android Application and Touch Pattern

\section{PROPOSED WORK}

The main objective is to automate lock system that can be operated through Android Application and Secured Touch patterns and to provide sharing facility to the user which could be implemented in many applications like smart mail box and vehicles as shown in figure 1 and 2 .

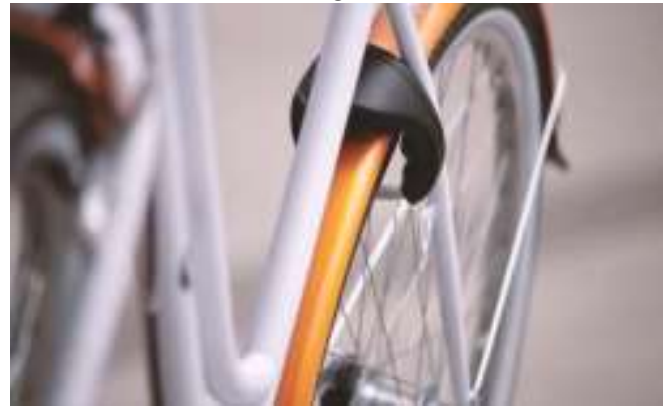

Fig.1 Vehicle Locker

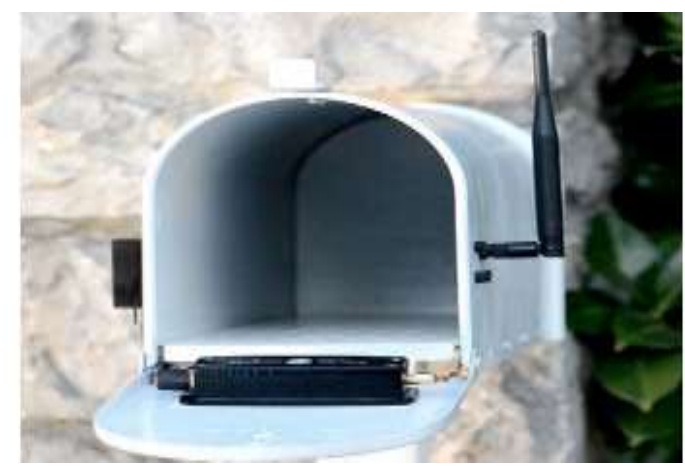

Fig.2 Mailbox Locker

The proposed work "Locker" provides an easy way of locking automobiles and for Mail Box locking system. The proposed work allows the user to lock and unlock easily and securely. Using one's phone, the lock system can be locked/unlocked by entering a pass code in the Android Application. If one forgets to bring the phone, he/she can use the system using the registered touch patterns that can be entered through a touch sensor present in the lock. It provides intelligent lock/unlock with the location of your phone to the locker with the Bluetooth. The user can share his/her pass key to allow his/her friends to use the lock
- Alarm and Monitoring

system and the shared code can only be used for a day which is regenerated every day. The system is powered by solar and so no external power supply is required. When anyone try to break the lock, the signal from the vibrate sensor facilitates the user to receive alert and "beep" sound continuously at the lock also. Thus, the system provides a convenient and smart way of locking system.

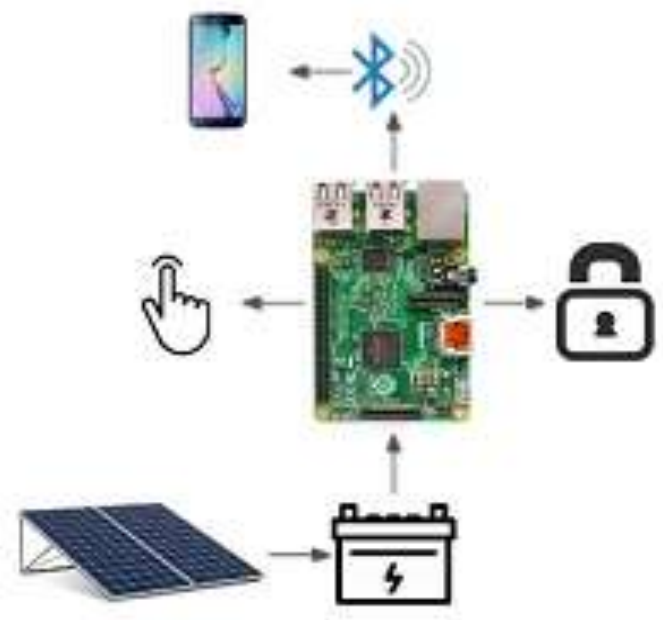

Fig.3 Proposed System

The prototype was developed using Raspberry Pi, Tap Sensor, Servo Motor, Beeper, Bluetooth and Solar panel. In the proposed work, the device "Locker" can be operated manually or remotely using Smartphone.

\section{Manual Operation}

\section{Unlocking}

1. The registered pattern(for ex:11110000) is used to unlock the device

2. While using the above pattern, $90^{\circ}$ rotation is induced the Servomotor and the device gets unlocked.

\section{Locking}

1. The registered pattern is used for locking the device

2. While using the above pattern, $90^{\circ}$ rotation in the opposite direction is induced in the Servomotor and the device gets locked.

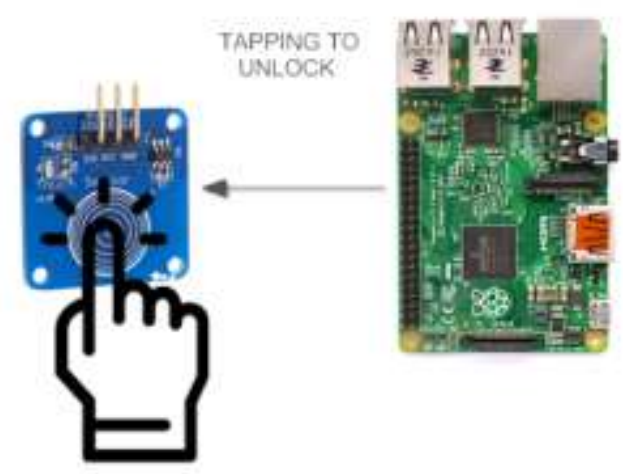

Fig.4 Manual Lock/Unlock 


\section{Using Android Application}

The Android APPLICATION is created for Remote operations either through Bluetooth of through Internet. If Bluetooth option is selected in the APPLICATION, then go to Lock or Unlock option as the case may be. For accessing through the Internet the Device IP address is selected and then goes to Lock or Unlock option.

LOCKIUNLOCK
USING
BLUETOOTH
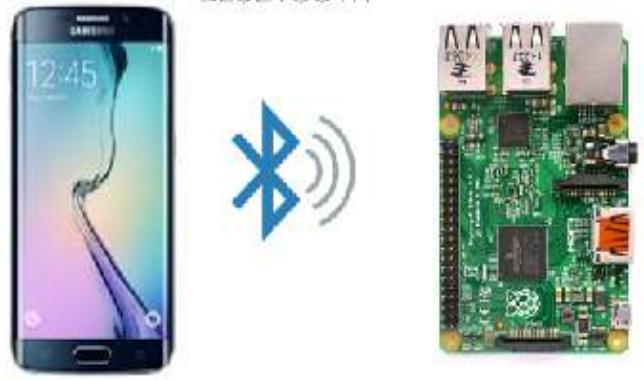

Fig.5 Lock/unlock using mobile application

\section{Theft Alarm}

The threshold value adopted for inducing alarm in the vibrating sensor is 5. Beep sound will be induced if the device gets vibrated more than this value. Simultaneously message alert will be received in the Smartphone.

\section{RESULTS AND DISCUSSIONS}

The sample results are discussed in this section.

The figure 6 and 7 shows the procedure to unlock/lock the device manually. If the registered pattern is entered matched, the device is locked/unlocked.

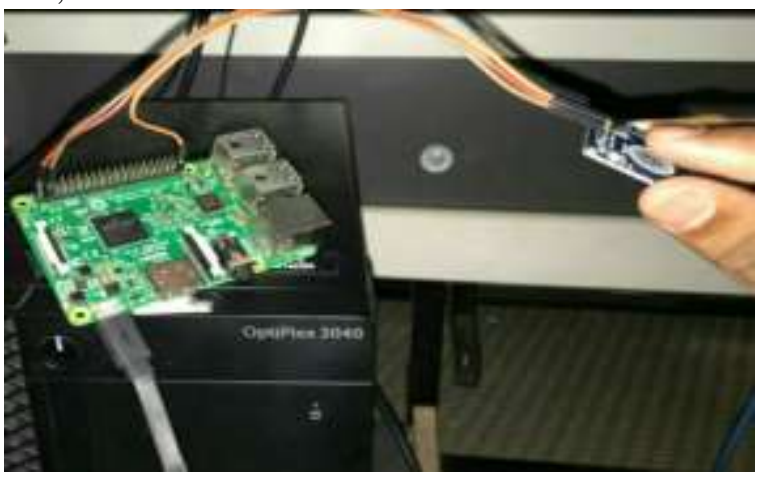

Fig.6 Unlock using tap sensor

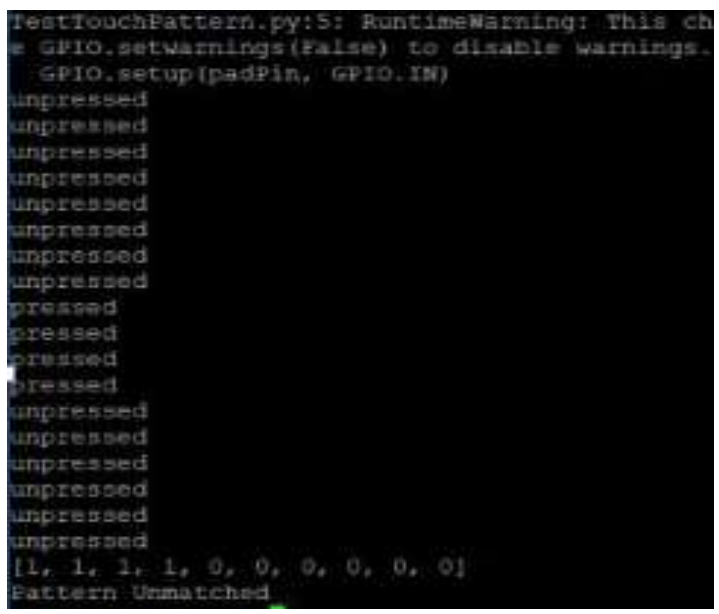

Fig.7 Unlock result
Figure 7 represents the sample error message where the pattern is not matched, so it says pattern unmatched.

Figure 8 is the mobile application which was developed to control the lock remotely using Bluetooth or Internet.

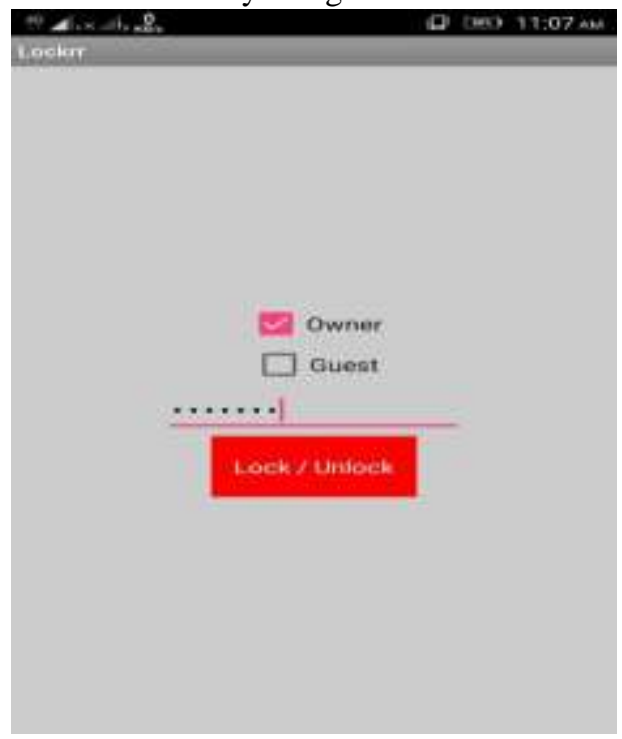

Fig.8 Mobile Application-Login

As already, the locker system also provides theft alarm message. Here is the sample alert message received.

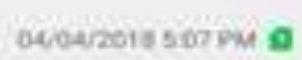 \\ Locker Alarm \\ + Hype message \\ Fig.9 Theft Alarm message \\ V. CONCLUSION}

We have identified two different application for this locker system. One is vehicle and the other is mail box. The Vehicle locker eliminates both the need to carry around a key, and the need to carry around a lock as well. Anytime one can walk away from the vehicle, it'll lock itself, and when walk towards it, it will be unlocked and ready for your ride. If someone attempt to steal your vehicle, Locker will sound an alarm, and send a smartphone notification alert to the owner.

The smart mailbox provides two features. First, it sends the notification to the user when the mail (letter/packages) has arrived to your door. Second, it allows the user to lock and unlock the mailbox remotely. It provides safe delivery for important packages or letters. The mailbox is connected to a home network through $\mathrm{Wi}-\mathrm{Fi}$. The mailbox is powered with solar energy. A battery is also on board, which will provide sufficient power in cloudy days.

The proposed system can also be implemented in industrial and commercial applications such as offices, warehouses, reserved areas where only authorized personnel

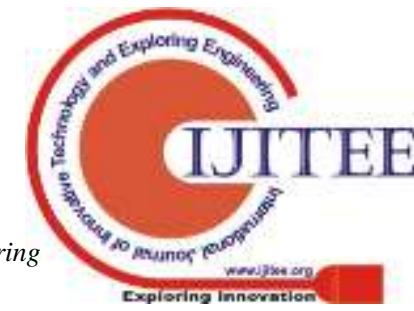


is allowed for entry and also places where safety and precautions are of primary concerns.

\section{REFERENCES}

1. PrashantkumarR.Sagar V. C Santosh S., SiddharthNambiar,Two Wheeler Vehicle Security System, International Journal of Engineering Sciences \& Emerging Technologies, Volume 6, Issue 3, pp: 324-334, Dec. 2013.

2. ArgadeGeetanjali Arjun, MoreshMukhedkar, "Advance Bike Security System" International Journal Of Science \&Research,Vol. 3, Issue 12,Dec 2014.

3. Nitin Kumar, Jatin Aggarwal, ChaviSachdeva, Prerna Sharma, Monica Gaur,International Journal of Education and Science Research,Volume-2, Issue - 2, April 2015

4. Pritpal Singh, TanjotSethi, Bibhuti Bhusan Biswal, and Sujit Kumar Pattanaya K, A Smart Anti-theft System for Vehicle Security, International Journal of Materials, Mechanics and Manufacturing, Vol. 3, No. 4, November 2015

5. Nagaraj, B., and P. Vijayakumar. "Soft computing based PID controller tuning and application to the pulp and paper industry." Sensors \& Transducers 133, no. 10 (2011): 30.

6. Umesh A. Patil, Pavan D. Gosavi, Sumit S. Chavan, ShubhamR.Jadhav, Nilam M. Pawar, "Secure Authentication System for Bike through Integrated Bluetooth Module",International Journal of Innovative Research in Computer and Communication Engineering (An ISO 3297: 2007 Certified Organization) Vol. 4 , Issue 2, February 2016.

7. K. Dinesh Kumar, B. Sasidharan, B. SruthiKeerthana, N. Tamil Selvan, N. L. Sivasundharam, "Password Based Lock for Bike Security with Ignition Key Control System", IJSART - Volume 2 Issue 5, May 2016.

8. Anitha .A, "Home security system using internet of things",IOP Conf. Series: Materials Science and Engineering, 263, 2017. 